\title{
Transition to Community Living After Acquired Brain Injury
}

\author{
Louise Gustafsson ${ }^{1}$ and Jennifer Fleming ${ }^{1,2,3}$ \\ ${ }^{1}$ School of Health and Rehabilitation Sciences, The University of Queensland, Australia \\ ${ }^{2}$ Occupational Therapy Department, Princess Alexandra Hospital, Queensland, Australia \\ ${ }^{3}$ Centre for Functioning and Health Research, Metro South Health Services District, Queensland, Australia
}

Enter the word 'transition' into an internet search engine and you will be overwhelmed by the number of web pages that talk about transitions from an economical, scientific, literary or organisational perspective (just to name a few). Common to the description of transition from the differing perspectives is the inclusion of the terms movement and/or change from one state to the next. It is important that healthcare professionals who work with people with both traumatic and nontraumatic brain injury appreciate and understand that from the point of brain insult, the patient and their family will experience multiple transitions involving movement or change in 'state', such as changes to roles and altering levels of participation and activity.

During the hospital stay, the patient and the family are supported by an intensive healthcare team who assist them with negotiating the new set of circumstances and beginning their adjustment to the changing states. At this time, the focus may be on basic healthcare and impairments of body structures and functions. Transition may be experienced in the form of moving from intensive care to an acute ward and then to subacute care in a rehabilitation unit. With each move, the patient and their family experience the transition to differing levels of care and changing expectations in terms of their abilities and participation in self-caring and rehabilitation activities. With each change there may be increasing awareness of the impact of the event on life outside of the hospital environment; however, this may not be truly actualised until after discharge from inpatient care. It is the transition to home and then to community living that is the focus of this special issue for Brain Impairment.

Transition to community living may include efforts to resume roles and activities within the home and in the community. It has been stated that people with stroke are often poorly prepared for the emotional and physical impacts of life at home and the community (National Stroke Foundation [NSF], 2007). Transition has also been described as an exciting yet stressful time for individuals with other forms of acquired brain injury and their families (Turner et al., 2007). The articles presented in this special issue on transition to community living bring together a series of quantitative and qualitative studies examining this process. In doing so, the diverse nature of the experience of transition and the importance of environmental factors such as support and service access are highlighted.

Transition to community living after acquired brain injury (ABI) is a process that primarily involves the resumption of participation in life roles. Since its introduction by the World Health Health Organization in 2001, the International Classification of Functioning, Disability and Health (ICF) has been used to conceptualise the process of transition to the community and participation is considered a primary goal of rehabilitation. This special issue on transition begins with an examination of the components of ICF in the context of participation after stroke by Hoyle, Gustafsson, Meredith and Ownsworth. In particular, they highlight the importance of contextual factors, including environmental and personal factors and their interactions with participation. This conceptual article throws light on the emerging importance of theories relating to personal factors such as biological disruption, self-discrepancy theory and threat appraisal and encourages the consideration of these factors within the ICF.

The following three articles describe studies using quantitative approaches to examine participation outcomes during the transition phase. Wolf, Brey, Baum and Tabor report the results of a study comparing the participation levels of younger and older people 6 months poststroke. Their findings 
demonstrate that younger people have different patterns of activity, both in terms of greater quantity and more active nature of activities both before and after stroke compared to older people. This has important implications for the types of activities offered in rehabilitation that traditionally focuses on basic activities of daily living. The authors conclude that for younger people with stroke, the focus of rehabilitation should shift to more high-demand activities such as work, accessing the community, leisure and fitness to facilitate transition to community living.

In the third article, Sloan, Callaway, Winkler and McKinley take a more long-term view of transition with a sample of individuals with severe to extremely severe $\mathrm{ABI}$ receiving a Community Approach to Participation (CAP) program over a 3 -year period. Using a longitudinal design, they compare the levels of independence, hours of support provided and participation outcomes of people residing in home-like settings with those in disability-specific facilities, demonstrating the importance of this environmental factor. In particular, the subgroup of participants who experienced an accommodation transition during the intervention period are described, illustrating that it is possible to achieve transitions to more home-like environments at late stages post-injury and that this is associated with increased independence and participation and a reduction in total care provided.

The influence of environment and service models is also the focus of the next article by Hopman, Tate and McCluskey comparing two different community-based rehabilitation programs for people with acquired brain injury. This longitudinal study compared the effectiveness of a transitional living unit program with a home-based program over a 6-month period. Both groups showed improvements in function and participation. Interestingly, outcomes did not differ greatly between the groups despite the transitional living program providing five times more intervention than the homebased program. In addition, this article provides a useful review of trials examining the effectiveness of community-based rehabilitation.

In another study examining service models, Kennedy, Barnes, Veitch and Rose describe the introduction of a new model of case management in an inpatient brain injury rehabilitation unit to facilitate transition to the community. Drawing on clinician perspectives gathered from semistructured interviews, this preliminary study suggests that early provision of coordinated comprehensive case management may facilitate a more streamlined and seamless transition home on discharge. Given that transition is a multifaceted process and a relatively new concept in the rehabilitation litera- ture, it makes sense to try and understand transition using qualitative methodologies.

The remaining articles in this special issue report on the results of qualitative research examining different aspects of transition from the perspectives of individuals with $\mathrm{ABI}$ and their caregivers. Collectively, they reveal a common thread suggesting that transitions from hospital to home and community life and work are characterised by focus on return to normality, engagement in meaningful and valued occupations and reconstruction of a sense of self. There are two articles that examine the insider perspective of transition from hospital based on the perspective of people with ABI. In the first of these, Conneeley used a longitudinal phenomenological approach to understand the experience of transition over the first year following rehabilitation discharge for people with severe traumatic brain injury. She provides a comprehensive examination of transition over three interview time points and from the perspective of individuals with brain injury, family members and health professionals. In the second article, Hall and colleagues used a mixed methods approach to explore the experience of transition to community living for a sample of working-aged people with nontraumatic brain injury. They found that key life circumstances influenced the transition experience. The findings support the need for individualised and structured transition services both before and after discharge for this group.

The third qualitative article explores transition back to work from the perspective of a young stroke survivor. Gustafsson and Turpin present a thematic analysis of an e-mail narrative over a 3-year period in which an individual with mild stroke describes her experiences while transitioning back to work. The findings demonstrate that the hidden impairments of stroke can greatly impact identity, including the struggle to reconcile a past worker identity with the present identity. The impact of fatigue on performance in work and nonwork time was highlighted as an important issue for consideration in any return-to-work program. The final qualitative study in this special issue is devoted to understanding the transition experience of family caregivers of people with traumatic brain injury. As illustrated by Nalder, Fleming, Cornwell and Foster, the lives of family members are inextricably linked with those of the injured individuals during the period of transition back to community living. Family members in this study described feeling the weight of their caring responsibility, leading to a desire for the family to move past the injury and return to normality. Embedded within the findings of this study and those of others in this special issue, are messages for clinicians working in brain 
injury rehabilitation. Understanding the meaning attributed to the transition phase by those experiencing it firsthand may assist service providers to develop intervention approaches that are more client-centred, contextually relevant and sensitive to the issues of self-identity that characterise the transition phase.

\section{References}

National Stroke Foundation. (2007). Walk in our shoes. Stroke survivors and carers report on support after stroke [Online]. Retrieved from http://www. strokefoundation.com.au/component/option,com_ docman/Itemid,39/task,cat_view/gid,61/

Turner, B., Fleming, J., Worrall, L., Cornwell, P., Haines, T., Ownsworth, T., Kendall, M., \& Chenoweth, L. (2007). A qualitative study of the transition from hospital to home for individuals with acquired brain injury and their family caregivers. Brain Injury, 21, 1119-1130.

World Health Organization. (2001). International Classification of Functioning, Disability and Health: ICF. Geneva: Author. 